DOI: https://doi.org/10.11144/Javeriana.upsy17-4.apat

\title{
Análisis psicométrico y adaptación del test Autoconcepto Forma 5 hacia el judo en una muestra chilena*
}

\section{Psychometric Analysis and Adaptation of the Questionnaire AF5 towards Judo in a Chilean Sample}

Recepción: 19 Marzo 2016 | Aceptación: 16 Septiembre 2017

\author{
Félix Zurita Ortega \\ Universidad de Granada, España \\ ORCID: http://orcid.org/0000-0002-1189-894X \\ Carmen González González de Mesa \\ Universidad de Oviedo, España \\ ORCID: http://orcid.org/0000-0001-8349-7494 \\ Asunción Martínez Martínez \\ Universidad de Granada, España \\ ORCID: http://orcid.org/0000-0002-8826-235X \\ Edson Orlando Zafra Santos \\ Universidad de Santo Tomás, Chile \\ ORCID: http://orcid.org/0000-0001-9583-1373 \\ Pedro Ángel Valdivia Moral \\ Universidad de Granada, España \\ ORCID: http://orcid.org/0000-0002-1905-3247
}

\footnotetext{
a Autor de correspondencia. Correo electrónico: asuncionmm@ugr.es
}

Para citar este artículo: Zurita Ortega, F., González González de Mesa, C., Martínez Martínez, A., Zafra Santos, E. O., \& Valdivia Moral, P. A. (2018). Análisis psicométrico y adaptación del test de Autoconcepto Forma 5 hacia el judo en una muestra chilena. Universitas Psychologica, 17(4), 1-10. https://doi.org/ 10.11144/Javeriana.upsy17-4.apat

\section{RESUMEN}

El objetivo del trabajo es analizar las propiedades psicométricas del cuestionario AF-5 propuesto por García y Musitu (1999), mediante técnicas de tipo exploratorio en judocas de Chile. La muestra de este estudio se encuentra conformada por 148 judocas con rango de edad entre 18 y 40 años, donde 86 participantes fueron hombres y 62 mujeres. El análisis de tipo factorial exploratorio fue llevado a cabo por el programa estadístico FACTOR Analysis. En cuanto a los resultados, estos indicaron que el test propuesto señalaba índices de fiabilidad superiores a $\alpha=0.7$. El análisis exploratorio no se ajusta al modelo pentafactorial original, eliminándose el factor familiar. En casi todos los factores, se encuentra correlación significativa en un valor de $99 \%(p<0.01)$, siendo el valor más alto el establecido al relacionarse la dimensión emocional con la física (0.521).

Palabras clave

Autoconcepto; judo; actividad física; deporte; propiedades psicométricas.

\begin{abstract}
The objective of this research was to analyze the psychometric properties of the questionnaire AF-5 (García \& Musitu, 1999) by exploratory techniques in a population of judokas of Chile. The sample consists of 148 judokas from 18 to 40 years, with 86 were men and the remaining 62 women. Exploratory factor analysis was performed using Factor Analysis program. The results indicate that the AF5 questionnaire provides higher
\end{abstract}


Félix Zurita Ortega, Carmen González González de Mesa, Asunción Martínez Martínez, et al.

reliability indexes $\alpha=0.7$. Referring to factorialidad exploratory analysis does not conform to the original fivefactor model eliminating the family factor. By applying the Pearson correlation coefficient, it shows that almost all significant correlation factors present in $99 \%(p<$ $0.01)$, the highest value established to relate the emotional dimension with the physical dimension (0.521).

Keywords

self-concept; judo; physical activity; sport; properties psychometric.

Las artes marciales y concretamente el judo está experimentando en los últimos años un auge considerable a nivel mundial; este aumento es muy notorio en Chile, donde la creación de espacios e infraestructuras está potenciando la realización de esta especialidad deportiva. Cada vez se tiende a conocer más de la práctica del deporte del judo, partiendo de las características de tipo físico, técnico y táctico que convergen y se abordan en esta modalidad, si se asemeja o compara con otras especialidades deportivas, aparte de constituir la base disciplinar y la capacidad de socialización que tiene.

Es una cuestión analizada y trabajada por numerosos estudios que lo abordan desde el prisma técnico-táctico (Drigo, Neto, Cesana, \& Tojal, 2011; Dopico et al., 2014), desde el análisis de la biomecánica o fisiología (Escobar, Rodríguez, Gutiérrez, \& Franchini, 2015; Malá et al., 2015), psicológico (Ziv \& Lidor, 2013; Vertonghen, Theeboom, \& Pieter, 2014; Kavoura, Ryba, \& Chroni, 2015) o de las lesiones deportivas (Kim, Park, Lee, \& Kang, 2015).

Desde el punto de vista del bienestar personal que genera esta especialidad, es el término de autoconcepto el que delimita y define las características personales que un individuo se atribuye y que se encuentra relacionado con parámetros de tipo físico, de comportamiento y emocional, tal y como plantean GarcíaSánchez, Burgueño, López y Ortega (2013). Esto se encuentra en concordancia con el modelo planteado por Shavelson, Hubner y Stanton (1976), que era de tipo jerárquico y que ha sido el más planteado en todos los ámbitos, pero desde que Wylie (1979) criticara la concepción unidimensional, la gran mayoría de autores consideran este constructo de forma multidimensional (Esnaola, Rodríguez, \& Goñi, 2011).

Algunos trabajos y estudios han manifestado una estrecha relación entre los tres parámetros objetos principales de este trabajo, que son el autoconcepto, el bienestar de tipo psicológico y la realización habitual de actividad física, como indican Goñi e Infante (2010) o Murgui, García, García y García (2012), mientras que otros trabajos han centrado su interés exclusivamente en el autoconcepto (Fox, 1988; Fox \& Corbin, 1989; Harter, 1982, 1985, 1993; Marsh, 1990; Standage, Duda, \& Ntoumanis, 2005; Standage $\&$ Treasure, 2002).

También se ha producido en los últimos años la adaptación del Test de Autoconcepto Forma 5 (AF5) a numerosos colectivos (Esnaola, 2005; García, Musitu, \& Veiga, 2006; Fernández, Contreras, García, \& González, 2010; García, Gracia, \& Zeleznova, 2013; Núñez, Martín, Navarro, \& Grijalvo, 2007; Tomás \& Oliver, 2004), pero son escasos los que la han empleado en la población chilena y los que lo han hecho enfocados hacia universitarios (Lobos, Díaz, Bustos, \& Pérez, 2015). Más recientemente, Fernández-Zabala, Rodríguez y Goñi (2016) citan la segmentación del cuestionario, trabajando concretamente el aspecto social. Otros autores como Goñi, Madariaga, Axpe y Goñi (2011) o el estudio planteado por Zurita, Castro, Álvaro, Rodríguez y Pérez (2016) hablan exclusivamente de la dimensión física.

No se encontró mucha información relacionada con las artes marciales y el autoconcepto, y en consecuencia se encuentra en ésta área un campo fértil para la investigación, acerca del cual discurre el presente documento, puesto que tal y como se está explicando la práctica del judo como una actividad de índole física interviene de forma directa sobre el autoconcepto y las dimensiones que lo componen. Debemos señalar que autores como Tomás y Oliver (2004), Núñez et al. (2007) o Esnaola et al. (2011) indican cómo se eliminaron factores en sus validaciones.

Por tanto, parece que el modelo de cinco factores no ha sido probado suficientemente en deportistas ni en personas chilenas mediante 
técnicas exploratorias, por lo que se plantea este estudio con el objetivo de analizar las propiedades psicométricas del AF5, con la finalidad de calcular la fiabilidad del instrumento para su adaptación y aplicación en una población de judocas chilenos. Para ello, se ha realizado un análisis factorial exploratorio (AFE).

\section{Método}

\section{Participantes}

En este trabajo de investigación de diseño descriptivo, participaron 148 judocas chilenos entre 18 y 40 años $(M=23.09$ años; $D E=$ 6.731), divididos en $n=86$ hombres $(58.1 \%)$ y $n=62$ mujeres (41.9\%), que se seleccionaron a través de un muestreo estratificado siguiendo las aportaciones de Santos, Muñoz, Juez y Cortiñas (2003). Según el nivel competitivo la muestra queda dividida en tres niveles: 58 profesionales (39.2\%), 47 amateur (31.8\%) y 43 aficionados (29.1\%). En la muestra, se aplican los cuestionarios a siete clubes de judo de Santiago de Chile que respondieron a instancias de los datos reportados por la Federación Chilena de Judo. También se suministraron a algunos de estos deportistas que no estaban afiliados a ningún club. Se debe señalar que durante la recogida y análisis de los datos se detectaron 18 cuestionarios erróneos por lo que se excluyeron del estudio; del mismo modo 23 judocas menores de edad no remitieron en tiempo y forma el correspondiente consentimiento informado de sus representantes legales, por lo que no formaron parte del estudio. La población objeto de estudio y sus respectivos datos sociodemográficos se puede observar en la siguiente tabla:

\section{Tabla 1}

Distribución de la muestra y datos de carácter sociodemográfico

\begin{tabular}{|c|c|c|c|c|c|c|}
\hline & & & Profesional & Amateur & Aficionado & Total \\
\hline \multirow{2}{*}{ Total } & & $\mathbf{N}$ & 58 & 47 & 43 & 148 \\
\hline & & $\%$ & (39.2) & $(31.8)$ & (29.1) & 100 \\
\hline \multirow{4}{*}{ Sexo } & & $N$ & 45 & 26 & 15 & 86 \\
\hline & Masculino & $\%$ & $(52.3)$ & $(30.2)$ & (17.4) & $(58.1)$ \\
\hline & & $\mathbf{N}$ & 13 & 21 & 28 & 62 \\
\hline & Femenino & $\%$ & (21.0) & (33.9) & $(45.2)$ & (41.9) \\
\hline
\end{tabular}

\section{Instrumentos}

El instrumento empleado fue el test Autoconcepto Forma-5 (AF-5) de García y Musitu (1999), que se fundamenta en el modelo teórico planteado por Shavelson et al. (1976). Está constituido por 30 preguntas cerradas agrupadas en cinco dimensiones: académica, social, emocional, familiar y física. La académica está conformada por las preguntas 1, 6, 11, 16, 21 y 26; la social, por los ítems 2, 7, 12, 17, 22 y 27; la de carácter emocional, por las cuestiones $3,8,13,18,23$ y 28 ; la familiar, por las cuestiones 4, 9, 14, 19, 24 y 29 y, finalmente, la física, por los ítems 5, 10, 15, 20, 25 y 30. En este instrumento, los participantes puntúan en una escala Likert del 1 al 5, donde el 1 es nunca hasta el 5 siempre. Esta forma de respuesta ha sido empleada y aceptada en otros estudios de similares características como el de Salum-Fares, Marín y Reyes (2011), aunque en su origen este cuestionario oscilaba entre los 1 y 99 puntos.

\section{Procedimiento}

Los cuestionarios se administraron en horario de no entrenamiento y en un periodo donde no se estuviese compitiendo y fue suministrado por el equipo investigador, que se preparó para este trabajo una vez consultados los deportistas y entrenadores.

Para los judocas menores de edad, les fue requerido el consentimiento informado, garantizándose en todos los casos que la información sería anónima e indicándose que solamente se utilizaría con fines de carácter científico. Como para cualquier trabajo de tipo científico, se obtuvo el permiso del Comité de Ética, en este caso proveniente de la Universidad de Santo Tomás de Chile (CE UST N.o 80/2014). Las personas analizadas desconocían la finalidad del estudio, con lo cual se evitaron las respuestas no sinceras y se redujo así al máximo el efecto de la deseabilidad social. 
Félix Zurita Ortega, Carmen González González de Mesa, Asunción Martínez Martínez, et al.

Análisis de los datos

Para el análisis de las propiedades psicométricas (fiabilidad y AFE), se empleó el paquete estadístico SPSS 24.0 para Windows y el Programa Factor Analysis 9.3.1 (Lorenzo-Seva $\&$ Ferrando, 2006). Tal como se utiliza en este tipo de estudios, en primer lugar se empleó el SPSS 24.0 para el análisis de las propiedades métricas de cada uno de los ítems del cuestionario y los coeficientes descriptivos básicos (media, dispersión, curtosis y asimetría). Después se exploró y utilizó el FACTOR, donde el diagnóstico de la bondad de ajuste es primordial para establecer la validez de la escala. La evaluación se fundamentó en los criterios recomendados por Bentler (1990) y McDonald y Marsh (1990). Finalmente y con el objetivo de verificar y establecer la consistencia interna del instrumento y de cada una de sus dimensiones, se empleó el coeficiente alpha de Cronbach.

\section{Resultados}

En esta primera parte del análisis y siguiendo las pautas recomendadas por Schmider, Ziegler, Danay, Beyer y Buhner (2010), se determinaron los valores descriptivos del instrumento. Se decidió eliminar cinco variables (V.14, V.17, V.19, V.24 y V.29) que alcanzaron valores superiores a 2 en las pruebas de dispersión (asimetría y curtosis).
Tabla 2

Valores descriptivos de los ítems de AF-5

\begin{tabular}{ccccccc}
\hline Ítems & Media & $\boldsymbol{D E}$ & Varianza & Asimetría & Curtosis & Rango \\
\hline V 01 & 3.81 & 0.971 & 0.944 & -1.191 & 1.572 & 4 \\
V 02 & 3.65 & 1.093 & 1.195 & -0.245 & -0.997 & 4 \\
V 03 & 3.28 & 1.002 & 1.004 & 0.076 & -0.493 & 4 \\
V 04 & 4.15 & 0.928 & 0.862 & -1.129 & 1.505 & 4 \\
V05 & 3.72 & 1.002 & 1.004 & -0.446 & -0.323 & 4 \\
V06 & 3.8 & 0.954 & 0.911 & -0.486 & -0.219 & 4 \\
V 07 & 4 & 0.94 & 0.884 & -0.896 & 0.508 & 4 \\
V 08 & 3.32 & 1.247 & 1.554 & -0.403 & -0.899 & 4 \\
V 09 & 4.3 & 0.965 & 0.931 & -1.089 & -0.08 & 3 \\
V 10 & 3.54 & 1.209 & 1.461 & -0.483 & -0.618 & 4 \\
V 11 & 3.28 & 1.101 & 1.211 & -0.336 & -0.355 & 4 \\
V 12 & 4.2 & 0.862 & 0.744 & -0.649 & -0.385 & 4 \\
V 13 & 3.94 & 1.038 & 1.078 & -0.801 & -0.038 & 4 \\
V 14 & 4.68 & 0.65 & 0.422 & -2.441 & 7.372 & 4 \\
V 15 & 3.27 & 1.007 & 1.015 & -0.283 & -0.206 & 4 \\
V 16 & 3.91 & 0.978 & 0.957 & -0.736 & 0.378 & 4 \\
V 17 & 4.32 & 0.793 & 0.629 & -1.314 & 2.158 & 4 \\
V 18 & 3.88 & 1.075 & 1.155 & -0.721 & -0.207 & 4 \\
V 19 & 4.66 & 0.743 & 0.552 & -2.29 & 4.494 & 3 \\
V 20 & 3.58 & 0.997 & 0.993 & -0.373 & -0.421 & 4 \\
V21 & 3.65 & 1.068 & 1.141 & -0.751 & 0.165 & 4 \\
V 22 & 4.11 & 1.064 & 1.131 & -1.182 & 1.089 & 4 \\
V 23 & 3.8 & 1.16 & 1.346 & -0.576 & -0.759 & 4 \\
V 24 & 4.48 & 0.861 & 0.741 & -1.882 & 3.529 & 4 \\
V 25 & 3.93 & 1.05 & 1.103 & -0.885 & 0.322 & 4 \\
V 26 & 3.89 & 0.915 & 0.837 & -0.526 & 0.016 & 4 \\
V 27 & 3.51 & 1.128 & 1.272 & -0.378 & -0.425 & 4 \\
V 28 & 3.91 & 1.026 & 1.052 & -0.728 & -0.089 & 4 \\
V 29 & 4.59 & 0.888 & 0.788 & -2.458 & 6.012 & 4 \\
V 30 & 3.55 & 1.026 & 1.052 & -0.493 & -0.113 & 4 \\
\hline & & & & & &
\end{tabular}

Previamente a la realización del AFE, se tomó la decisión de eliminar por completo la dimensión familiar, pues cuatro de sus variables tenían valores por encima de lo normal en la curtosis y la asimetría. También se prescindió de la variable 17 por presentar valores excesivamente altos en estas mismas pruebas de dispersión.

A continuación, y aplicándose el programa FACTOR, se rotaron cuatro factores. Los resultados establecieron un estadístico de Bartlett de 1672.1 ( $d f=253 ; p=0.000010)$ y un $\mathrm{KMO}$ (Test de Kaiser-Meyer-Olkin) de 0.77, que se emplea para determinar si la muestra viene o procede de poblaciones que tienen la misma varianza y que determinen si existe una buena adecuación muestral; en este sentido, y según los datos hallados, se debe señalar un ajuste aceptable de los datos para ser sometidos al análisis factorial.

Los cuatro factores extraídos explican el 58 $\%$ de la varianza total; en cuanto al índice de bondad de ajuste (GFI) el valor fue de 0.98 y la raíz cuadrática media de los residuales (RMSR) 
obtuvo una cifra de 0.05 , estos datos indican un buen ajuste para los ítems.

En los datos de la Tabla 3, se observa cómo se suprimieron dos variables (V.6 y V.22), puesto que cargaron en dos factores con un valor de diferencia de menos de 0.1 , por lo que la escala final quedó conformada por cuatro factores. El primer factor, que se corresponde con la dimensión emocional, está compuesto de seis variables; el Factor 2 se encuentra formado por cuatro variables que determinan la dimensión académica (se excluyó la V.16 pues cargó donde no debía, tal vez por una mala comprensión del ítem); en el tercer factor confluyen seis variables y se corresponde con la dimensión física y, por último, el cuarto factor constituido por cuatro variables determina la dimensión social.

\section{Tabla 3}

Matriz factorial rotada

\begin{tabular}{|c|c|c|c|c|c|c|c|c|}
\hline \multirow{2}{*}{ Variables } & \multicolumn{4}{|c|}{ Matriz Factorial Rotada Completa } & \multicolumn{4}{|c|}{ Cargas inferiores a 0.3} \\
\hline & F1 & F2 & F3 & $\mathrm{F} 4$ & F1 & F2 & F3 & F4 \\
\hline $\begin{array}{lll}\mathrm{V} & 01\end{array}$ & -0.022 & 0.82 & -0.148 & -0.028 & & 0.82 & & \\
\hline $\mathrm{V} \quad 02$ & -0.045 & 0.029 & 0.095 & 0.86 & & & & 0.86 \\
\hline V 03 & 0.672 & -0.225 & 0.025 & 0.064 & 0.672 & & & \\
\hline V 05 & 0.119 & 0.324 & 0.497 & -0.244 & & & 0.497 & \\
\hline V 06 & -0.042 & 0.47 & 0.543 & -0.094 & & & & \\
\hline V 07 & -0.141 & 0.263 & 0.08 & 0.537 & & & & 0.537 \\
\hline V 08 & 0.773 & -0.029 & 0.116 & -0.096 & 0.773 & & & \\
\hline V 10 & -0.067 & -0.142 & 0.73 & 0.218 & & & 0.73 & \\
\hline V 11 & -0.077 & 0.806 & 0.056 & -0.004 & & 0.806 & & \\
\hline V 12 & 0.28 & 0.148 & -0.33 & 0.607 & & & & 0.607 \\
\hline V 13 & 0.739 & -0.031 & -0.021 & 0.007 & 0.739 & & & \\
\hline V 15 & 0.221 & -0.13 & 0.476 & 0.044 & & & 0.476 & \\
\hline V 16 & -0.084 & 0.235 & 0.68 & -0.058 & & & 0.68 & \\
\hline V 18 & 0.643 & 0.019 & 0.124 & 0.017 & 0.643 & & & \\
\hline V 20 & 0.131 & 0.154 & 0.596 & -0.121 & & & 0.596 & \\
\hline V 21 & 0.023 & 0.845 & 0.001 & -0.021 & & 0.845 & & \\
\hline V 22 & 0.264 & 0.123 & -0.037 & 0.121 & & & & \\
\hline V 23 & 0.402 & 0.174 & 0.113 & 0.024 & 0.402 & & & \\
\hline V 25 & -0.102 & -0.108 & 0.808 & 0.067 & & & 0.808 & \\
\hline V 26 & 0.099 & 0.403 & 0.502 & -0.001 & & 0.403 & & \\
\hline V 27 & -0.032 & -0.121 & 0.171 & 0.473 & & & & 0.473 \\
\hline V 28 & 0.78 & 0.075 & -0.113 & -0.039 & 0.78 & & & \\
\hline V 30 & 0.072 & -0.22 & 0.51 & 0.106 & & & 0.51 & \\
\hline
\end{tabular}

Una vez determinados los factores, se establecieron la estructura y configuración del cuestionario con un buen coeficiente de fiabilidad $(p=0.849)$ y se eliminaron aquellas variables que no cargaban de manera normal; se obtuvieron los siguientes resultados: 0.84 para el Factor 1 (emocional), 0.794 para el Factor 2 (académico), 0.822 para el Factor 3 (físico) y 0.773 en el Factor 4 (social).
Tabla 4

Dimensiones del AF-5 y su carga factorial

\begin{tabular}{|c|c|c|c|c|}
\hline Variables & F1 & F2 & F3 & F4 \\
\hline V 03 & 0.672 & & & \\
\hline V 08 & 0.773 & & & \\
\hline V 13 & 0.739 & & & \\
\hline V 18 & 0.643 & & & \\
\hline V 23 & 0.402 & & & \\
\hline V 28 & 0.78 & & & \\
\hline V 01 & & 0.82 & & \\
\hline V 11 & & 0.806 & & \\
\hline V 21 & & 0.845 & & \\
\hline V 26 & & 0.403 & & \\
\hline V 05 & & & 0.497 & \\
\hline V 10 & & & 0.73 & \\
\hline V 15 & & & 0.476 & \\
\hline V 20 & & & 0.596 & \\
\hline V 25 & & & 0.808 & \\
\hline $\mathrm{V} \quad 30$ & & & 0.51 & \\
\hline V 02 & & & & 0.86 \\
\hline V 07 & & & & 0.537 \\
\hline V 12 & & & & 0.607 \\
\hline V 27 & & & & 0.473 \\
\hline alfa $(0.849)$ & 0.84 & 0.794 & 0.822 & 0.773 \\
\hline
\end{tabular}

Tal y como se establece en la Tabla 4, el coeficiente de fiabilidad alfa de Cronbach obtuvo valores muy satisfactorios, tanto en el cuestionario en general como en cada uno de los factores o dimensiones, con valores superiores a $p>0.7$. Una vez aplicado el coeficiente de correlación de Pearson, se determinó que en casi todas las dimensiones hay una correlación significativa al $99 \%(p<0.01)$, hallándose la cifra más alta al relacionarse la dimensión emocional con la dimensión física (0.521). También presentaron correlaciones significativas el factor académico con el físico y el social; asimismo, el factor físico también correlaciona significativamente, pero en menor medida $(p<$ 0.05), con la dimensión social. 
Tabla 5

Correlación de las dimensiones del AF5

\begin{tabular}{lcccc}
\hline \multicolumn{1}{c}{ Factor } & F1 & F2 & F3 & F4 \\
\hline Factor 1: Dimensión Emocional & 1 & & & \\
Factor 2: Dimensión Académico & 0.13 & 1 & & \\
Factor 3: Dimensión Físico & $0.52^{* *}$ & $0.21^{* *}$ & 1 & \\
Factor 4: Dimensión Social & 0.12 & $0.27^{* *}$ & $0.19^{*}$ & 1 \\
\hline Alf de Cronbach (Total cuestionario 0.84) & 0.84 & 0.79 & 0.82 & 0.77 \\
\hline \multicolumn{4}{c}{$*$ La correlación es significativa al } \\
\multicolumn{4}{c}{ nivel 0.01 (bilateral). * La correlación } \\
es significativa al nivel 0.05 (bilateral).
\end{tabular}

\section{Discusión}

Para establecer la evaluación psicológica en el contexto del deporte, es necesario tener herramientas e instrumentos que aporten datos y valores fiables de lo que se quiera analizar o medir, tal como sucedió con el objetivo de esta investigación que fue el de analizar las propiedades psicométricas del cuestionario AF-5 y establecer su adaptabilidad y aplicabilidad en la población de judocas chilenos.

El deporte objeto de estudio reúne unas características que lo diferencian del resto (Vertonghen et al., 2014) y, como plantean Peset et al. (2013), posee unas condiciones culturales muy afincadas desde hace siglos que lo hacen bastante arraigado en las tradiciones.

Los resultados obtenidos fueron los adecuados en lo que a los índices de fiabilidad analizados se refiere, obtenidos a través del alpha de Cronbach tanto en el general como en cada uno de los cuatro factores, constituyéndose en instrumento fiable y válido para establecer el autoconcepto de un modo multidimensional, incluso prescindiendo del factor familiar. Para comprender este concepto, se debe partir de las apreciaciones citadas por los autores GonzálezPienda et al. (2000) y Goñi y Fernández (2007), en lo referente a los efectos que tiene en el ámbito social, académico y emocional; en lo concerniente al familiar, este queda eliminado, mientras que en otros trabajos sí aparece al quedar demostrada la importancia de esta dimensión, cuando se estudia en los sujetos adolescentes, tal y como lo plantean Molero, Ortega-Álvarez, Valiente y ZagalazSánchez (2010).
Los datos obtenidos se mantienen estables en la dimensión emocional y física, cargando los factores de una forma coherente, mientras que en las dimensiones social y académica se pierde alguna variable. Si bien este test ha sido ampliamente aplicado en el contexto deportivo y de la práctica (Arostegi, Goñi, Zubillaga, \& Infante, 2013; Cachón-Zagalaz, Cuervo, Zagalaz, \& González, 2015; Murgui, García, \& García, 2016; Zurita et al., 2016), no lo había sido nunca en el judo.

Como se citó anteriormente, con la omisión del factor familiar, los valores obtenidos apoyan la teoría de la multidimensionalidad, presentando unos índices de ajuste muy aceptables con una excelente consistencia interna y una buena estabilidad temporal, ya que presenta valores superiores al 0.7 y refuta el modelo planteado por Shavelson et al. (1976) que ya se ajustó de manera coherente en los estudios de Tomás y Oliver (2004) y de Murgui et al. (2012); igualmente, establece una mayor perspectiva en comparación con las herramientas que solamente se centran en el apartado físico, tal y como indicaron Marsh, Bar-Eli, Zach y Richards (2006). Las cifras halladas sugieren que algunas de las cuestiones deberían ser desarrolladas y trabajadas en trabajos futuros.

La mayor correlación de este trabajo se encontró entre la dimensión física y emocional, entendiendo que puede estar generada por la especialidad deportiva objeto de estudio, la cual se caracteriza por concentración, capacidad de anticipación, estado de flow, control emocional, autocontrol, confianza y competitividad, entre otros (Anshel \& Payne, 2006; Crivelli, Carrera, \& Fernández, 2015). También se encontró correlación, aunque en menor medida, entre la dimensión física y la académica y social, pudiéndose deducir que al ser una modalidad individual se establece un menor componente social, por lo que los aspectos relacionales, como pueden ser el trabajo en equipo o la cooperación, se encuentran disminuidos en relación con otras especialidades deportivas (Vink, Raudsepp, \& Kais, 2014). En este sentido, Montero, Moreno, González y Cervelló (2013) mostraron en sus documentos cifras semejantes en las 
características de motivación, autoconfianza y flow, en judocas de alto nivel.

Tal y como sugieren en su trabajo García y Musitu (1999), todos los factores del test deberían reportar datos concisos sobre la percepción de sí mismos en personas jóvenes y adultas, sin embargo y a pesar de esta premisa, este estudio explica la exclusión de la dimensión familiar. En este sentido, el judo engloba numerosas características, no encontrándose entre ellas la familiar, pues también los patrones de los judocas participantes en el estudio son diversos, dado el rango de edad de los 18 a los 40 años, donde pueden encontrarse situaciones familiares muy diversas.

Por lo tanto, es probable que los factores físicos y emocionales se hallen incrementados respecto al componente social y académico, este último tal vez debido a la asociación con el nivel competitivo, que ya fue analizado por Armstrong y Oomen (2009), por lo que un mayor componente cognitivo podría predominar sobre la capacidad motriz, e igualmente, es lógico pensar que la dimensión emocional afecta al nivel físico, sobre todo en el ámbito de la competencia.

Desde la perspectiva metodológica, este modelo apoya el uso de la medida global del autoconcepto hacia las artes marciales como actividad deportiva, si bien presenta algunas limitaciones como ha sido la no probabilística $\mathrm{y}$, por tanto, los resultados no pueden ser generalizados a toda la población chilena. Además, en futuros trabajos debería explorarse la estructura de otras artes marciales (taekwondo, kárate,...). En segundo lugar, se podrían utilizar muestras comparativas para determinar posibles diferencias con otras variables (p. ej., género, logros deportivos, nivel educativo). En resumen, podría decirse que los resultados de este trabajo apoyan la idea del AF5 como test válido y fiable para medir de forma satisfactoria el autoconcepto en judocas y su análisis multidimensional (excluyendo el componente familiar).

\section{Referencias}

Anshel, M., \& Payne, J. (2006). Application of sport psychology for optimal performance in martial arts. En J. Dosil (Ed.), The sport psychologist's handbook (pp. 353-374). Chichester, UK: John Wiley.

Armstrong, S., \& Oomen, J. (2009). Social connectedness, self-esteem and depression symptomatology among collegiate athletes versus non-athletes. Journal of American College Health, 57(5), 521-526. https://doi. org/10.3200/JACH.57.5.521-526

Arostegi, B., Goñi, A., Zubillaga, A., \& Infante, G. (2013). El autoconcepto físico de jóvenes futbolistas de alto rendimiento. Cuadernos de Psicología del Deporte, 13(1), 9-14. https://doi.org/10.4321/S1578-84232 013000100002

Bentler, P. M. (1990). Comparative fit indexes in structural models. Psychological Bulletin, 107, 238-246. http://dx.doi.org/10.1037/00 33-2909.107.2.238

Cachón-Zagalaz, J., Cuervo, C., Zagalaz, M. L., \& González, C. (2015). Relación entre la práctica deportiva y las dimensiones del autoconcepto en función del género y la especialidad que cursan los estudiantes de los grados de magisterio. Journal of Sport and Health Research, 7(3), 257-266. Recuperado de https://www.researchgate.net/publicatio $\mathrm{n} / 281589179$

Crivelli, C., Carrera, P., \& Fernández, J. M. (2015). Are smiles a sign of happiness? Spontaneous expressions of judo winners. Evolution and Human Behavior, 36, 52-58. https://doi.org/10.1016/j.evolhumbe hav.2014.08.009

Dopico, X., Iglesias, E., Carballeira, E., Mayo, X., Ardá, A., \& González, M. (2014). The relationship between motoric dominance and functional dominance while executing judo techniques: A study on laterality. Archives of Budo, 10, 325-331. Recuperado de http://archbudo.com/view/abstracts/issu e_id/387

Drigo, A., Neto, S., Cesana, J., \& Tojal, J. (2011). Artes marciais, formação profissional e 
Félix Zurita Ortega, Carmen González González de Mesa, Asunción Martínez Martínez, et al.

escolas de ofício: Análise documental do judô brasileiro. Motricidade, 7(4), 49-62. ht tps://doi.org/10.6063/motricidade.7(4).88

Escobar, R., Rodríguez, S., Gutiérrez, C., \& Franchini, E. (2015). Weight loss and psychological-related states in high-level judo athletes. International Journal of Sport Nutrition and Exercise Metabolism, 25(2), 110-118. https://doi.org/10.1123/ijsnem.20 13-0163

Esnaola, I. (2005). Autoconcepto físico y satisfacción corporal en mujeres adolescentes según el deporte practicado. Apunts. Educación Física y Deportes, 2(80), 5-12. Recuperado de https://www.raco.cat/index.php/Apunts EFD/article/view/300953/390399

Esnaola, I., Rodríguez, A., \& Goñi, E. (2011). Propiedades psicométricas del Cuestionario de Autoconcepto AF5. Anales de Psicología, 27(1), 109-117. Recuperado de http://www .redalyc.org/html/167/16717018013/

Fernández, J. G., Contreras, O., García, L. M., \& González, S. (2010). Autoconcepto físico según la actividad físico-deportiva realizada y la motivación hacia esta. Revista Latinoamericana de Psicología, 42 (2), 251-263. https://doi.org/10.14349/rlp.v42i 2.478

Fernández-Zabala, A., Rodríguez, A., \& Goñi, A. (2016). The structure of the Social Self Concept (SSC) Questionnaire. Anales de Psicología, 32(1), 199-205. https://doi.org/1 0.6018/analesps.32.1.193931

Fox, K. R. (1988). The self-esteem complex and youth fitness. Quest, 40, 230-246. https://research-information.brist ol.ac.uk/...selfesteem-complex-and-youth-f itness (74b76f06-82be-46d5-9401-fa743b9 7a129)/export.html

Fox, K. R., \& Corbin, C. B. (1989). The physical self-perception profile: Development and preliminary validation. Journal of Sports and Exercise Psychology, 11, 408-430. https://doi .org/10.1123/jsep.11.4.408

García, F., Gracia, E., \& Zeleznova, A. (2013). Validation of the English version of the Five-Factor Self-Concept Questionnaire.
Psicothema, 25(4), 549-555. https://doi.org/ 10.7334/psicothema2013.33

García, F., \& Musitu, G. (1999). AF5: Autoconcepto Forma 5. Madrid: TEA.

García, F., Musitu, G., \& Veiga, F. (2006). Autoconcepto en adultos de España y Portugal. Psicothema, 18(3), 551-556. Recuperado de http://www.psicothema.co $\mathrm{m} / \mathrm{psicothema} \cdot$ asp? $\mathrm{id}=3252$

García-Sánchez, A., Burgueño, R., López, D., \& Ortega, F. B. (2013). Condición física, adiposidad y autoconcepto en adolescentes. Estudio Piloto. Revista de Psicología del Deporte, 22(2), 453-461. Recuperado de http://www.redalyc.org/htm 1/2351/235128058014/

González-Pienda, J. A., Núñez, J. C., GonzálezPumariega, S., Álvarez, L., Roces, C., García, M., ... Valle, A. (2000). Autoconcepto, proceso de atribución causal y metas académicas en niños con o sin dificultades de aprendizaje. Psicothema, 12(4), 548-556. Recuperado de http://www.beatrizblancopsicologa.com/ pdfs/7759-13462-1-PB.pdf

Goñi, E., \& Fernández, A. (2007). Los dominios social y personal del autoconcepto. Revista de Psicodidáctica, 12 (2), 179-194. http://hdl .handle.net/10810/7098

Goñi, E., \& Infante, G. (2010). Actividad físico-deportiva, autoconcepto físico y satisfacción con la vida. European Journal of Education and Psychology, 3(2), 199-208. ht tps://doi.org/10.1989/ejep.v3i2.60

Goñi, E., Madariaga, J. M, Axpe, I., \& Goñi, A. (2011). Structure of the Personal Self-Concept (PSC) Questionnaire. International Journal of Clinical and Health Psychology, 11(3), 509-522. Recuperado de https://pdfs.semanticscholar.org/5a6c/bf fcab45045374b0a8cad5065a9a296049dc.p df

Harter, S. (1982). The Perceived Competence Scale for Children. Child Development, 53(1), 87-97. https://doi.org/10.2307/1129 640

Harter, S. (1985). Manual for the Self-Perception Profile for Children: Revision of the Perceived 
Competence Scale for Children. Denver, CO: University of Denver.

Harter, S. (1993). Causes and consequences of low self-esteem in children and adolescents. Nueva York: Springer.

Kavoura, A., Ryba, T.V., \& Chroni, S. (2015). Negotiating female judoka identities in Greece: A foucauldian discourse analysis. Psychology of Sport and Exercise, 17, 88-98. https://doi.org/10.1016/j.psychsport. 2014.09.011

Kim, K. S., Park, K. J., Lee, J. K., \& Kang, B. Y. (2015). Injuries in national Olympic level judo athletes: An epidemiological study. British Journal of Sports Medicine, 49(17), 1144-1150. https://doi.org/10.1136/bjsport s-2014-094365

Lobos, K., Díaz, A., Bustos, C., \& Pérez, M. V. (2015). Construction and psychometric characteristic of the Self-Concept Scale of Interaction in the Classroom. Psicothema, 27(2), 151-158. https://doi.org/10.7334/psi cothema2014.224

Lorenzo-Seva, U., \& Ferrando, P. J. (2006). FACTOR: A computer program to fit the exploratory factor analysis model. Behavioral Research Methods, Instruments and Computers, 38(1), 88-91. https://doi.or $\mathrm{g} / 10.3758 / \mathrm{BF} 03192753$

Malá, L., Maly, T., Zahálka, F., Heller, J., Hrasky, P., \& Vodicka, P. (2015). Differences in the morphological and physiological characteristics of senior and junior elite Czech judo athletes. Archives of Budo, 11, 185-193. Recuperado de https:/www.researchgate.net/publicatio n/281121146_Differences_in_the_morpho logical

Marsh, H. W. (1990). The structure of academic self-concept: The Marsh/Shavelson model. Journal of Educational Psychology, 82, 623-636. https://doi.org/10.1037/0022-066 3.82.4.623

Marsh, H., Bar-Eli, M., Zach, S., \& Richards, G. (2006). Contruct validation of Hebrew versions of three physical selfconcept measures and extended multitraitmultimethod analysis. Journal of Sport and
Exercise Psychology, 28, 310-343. https://doi .org/10.1123/jsep.28.3.310

McDonald, R. P., \& Marsh, H. W. (1990). Choosing a multivariate model: Noncentrality and goodness of fit. Psychological Bulletin, 107, 247-255. https:// doi.org/10.1037/0033-2909.107.2.247

Molero, D., Ortega-Álvarez, F., Valiente, I., \& Zagalaz-Sánchez, M. L. (2010). Estudio comparativo del autoconcepto físico en adolescentes en función del género y del nivel de actividad físico-deportiva. RETOS. Nuevas Tendencias en Educación Física, Deporte y Recreación, 17, 38-41. Recuperado de http://www.redalyc.org/pdf/ 3457/345732283008.pdf

Montero, C., Moreno, J. A., González, D., \& Cervelló, E. (2013). Motivación, dirección de la autoconfianza y flow en judokas de alto nivel. Motricidad: European Journal of Human Movement, 31, 1-16. Recuperado de http://www.redalyc.org/html/2742/2742 29586001/

Murgui, S., García, C., García, A., \& García, F. (2012).Autoconcepto en jóvenes practicantes de danza y no practicantes: análisis factorial confirmatorio de la Escala AF5. Revista de Psicología del Deporte, 21 (2), 263-269. Recuperado de http://www.redaly c.org/pdf/2351/235126897006.pdf

Murgui, S., García, C., \& García, A. (2016). Efectos de la práctica deportiva en la relación entre las habilidades motoras, el autoconcepto físico y el autoconcepto multidimensional. Revista de Psicología del Deporte, 26(1), 19-25. Recuperado de http://www.redalyc.org/pdf/2351/23514 3645003.pdf

Núñez, J. L., Martín, J., Navarro, J. G., \& Grijalvo, F. (2007). Análisis de las propiedades psicométricas del cuestionario Autoconcepto Forma 5 en estudiantes universitarios. Estudios de Psicología, 28(3), 333-342. https://doi.org/10.1174/02109390 7782506461

Peset, F., Ferrer, A., Villamón, M., Millán, L., Toca, J. L., \& Aleixandre, R. (2013). Scientific literature analysis of judo in Web 
Félix Zurita Ortega, Carmen González González de Mesa, Asunción Martínez Martínez, et al.

of Science. Archives of Budo, 9(2), 81-91. Recuperado de http://eprints.rclis.org/2100 8/1/fulltext564.pdf

Salum-Fares, A., Marín, A. R., \& Reyes, A. C. (2011). Relevancia de las dimensiones del autoconcepto en estudiantes de escuelas secundarias de Ciudad Victoria, Tamaulipas, México. Revista Electrónica de Psicología Iztacala, 14(2), 255-272. Recuperado de http://www.medigraphic.co m/pdfs/epsicologia/epi-2011/epi112n.pdf

Santos, J., Muñoz, A., Juez, P., \& Cortiñas, P. (2003). Diseño de encuestas de estudio de mercado. Técnicas de muestreo y análisis multivariante. Madrid: Fundación Ramón Areces.

Schmider, E., Ziegler, M., Danay, E., Beyer, L., \& Bühner, M. (2010). Is it really robust? Reinvestigating the robustness of ANOVA against violations of the normal distribution assumption. Meythodology, 6, 147-151. http s://doi.org/10.1027/1614-2241/a000016

Shavelson, J., Hubner, J. J., \& Stanton, G. C. (1976). Self-concept: Validation of construct interpretations. Review of Educational Research, 46, 407-442. https://d oi.org/10.3102/00346543046003407

Standage, M., Duda, J. L., \& Ntoumanis, N. (2005). A test of self-determination theory in school physical education. British Journal of Educational Psychology, 75, 411-433. http s://doi.org/10.1348/000709904X22359

Standage, M., \& Treasure, D. C. (2002). Relationship among achievement goal orientations and multidimensional situational motivation in physical education. British Journal of Educational Psychology, 72(1), 87-103. https://doi.org/1 0.1348/000709902158784

Tomás, J. M., \& Oliver, A. (2004). Confirmatory factor analysis of a Spanish multidimensional scale of self-concept. Revista Interamericana de Psicología, 38(2), 285-293. Recuperado de https://dialnet.uni rioja.es/servlet/articulo?codigo $=3023239$

Vertonghen, J., Theeboom, M., \& Pieter, W. (2014). Mediating factors in martial arts and combat sports: An analysis of the type of martial art, characteristics, and social background of young participants. Perceptual and Motor Skills, 118(1), 41-61. https://doi.org/10.2466/06.30.PMS. $118 \mathrm{k} 14 \mathrm{w} 3$

Vink, K., Raudsepp, L., \& Kais, K. (2014). Intrinsic motivation and individual deliberate practice are reciprocally related: Evidence from a longitudinal study of adolescent team sport athletes. Psychology of Sport and Exercise, 16, 1-6. https://doi.org/1 0.1016/j.psychsport.2014.08.012

Wylie, R. C. (1979). The self-concept: Vol. 2. Theory and research on selected topics. Lincoln, NE: University of Nebraska.

Ziv, G., \& Lidor, R. (2013). Psychological preparation of competitive judokas - A review. Journal of Sports Science Eु Medicine, 12 (3), 371-380. https://doi.org/10.12800/cc d.v3i7.152

Zurita, F., Castro, M., Álvaro, I., Rodríguez, S., \& Pérez, A. (2016). Autoconcepto, actividad física y familia: un modelo de ecuaciones estructurales. Revista de Psicología del Deporte, 25(1), 97-104. Recuperado de https:/www.researchgate.net/publicatio n/294260938_Autoconcepto_Actividad_fi sica_y_Familia_Analisis_de_un_modelo_d e_ecuaciones_estructurales

\section{Notas}

* Artículo de investigación. 